\title{
Observation of Optical Transients and Search for PeV-EeV Tau Neutrinos with Ashra-1
}

\author{
Satoru Ogawa*, Hitoshi Oshima, Rikuto Nagasawa \\ Department of Physics, Toho University, Funabashi, Chiba 274-8510, Japan \\ E-mail: ogawa@ph.sci.toho-u.ac.jp \\ Makoto Sasaki, Toshifumi Aoki \\ Institute for Cosmic Ray Research, University of Tokyo, Kashiwa, Chiba 277-8582, Japan \\ E-mail: sasakimeicrr.u-tokyo.ac.jp
}

\section{other authors for the Ashra-I Collaboration ${ }^{\dagger}$}

\begin{abstract}
We report the updated observational search for tau neutrinos $\left(v_{\tau}\right)$ using one of the Ashra light collectors, which faces to Mauna Kea, on Mauna Loa at 3300 m above sea level on Hawaii Island. The earth-skimming $v_{\tau}$ technique of imaging Cherenkov $\tau$ showers was applied as a detection method. We checked the detection sensitivity and the reconstruction procedure for the Cherenkov $\tau$ shower well with sufficient statistics, i.e. 824 events of ordinary cosmic-ray airshower Cherenkov images for a total of 1884 hours using the same instruments, but triggered with the attached trigger pixel layout to view the sky field above the mountain. The analysis of about 1863 hours of data taken towards the mountain of Mauna Kea and the Earth crust around it leads to a $90 \%$ C.L. point source limit for $v_{\tau}$ in the energy range from $3 \mathrm{PeV}$ to $10 \mathrm{EeV}$ of about $\mathrm{E}_{v_{\tau}}^{2} \cdot \phi\left(\mathrm{E}_{v_{\tau}}\right)<3.2 \times 10^{-7} \mathrm{GeV} \mathrm{cm}^{-2} \mathrm{~s}^{-1}$ assuming power-law $v$ spectrum with the index of -2 . The analysis status of about 5783 hours of optical transients data is also presented.
\end{abstract}

36th International Cosmic Ray Conference -ICRC2019-

July 24th - August 1st, 2019

Madison, WI, U.S.A.

\footnotetext{
*Speaker.

${ }^{\dagger}$ for the collaboration list see PoS(ICRC2019)1177
} 


\section{Introduction}

IceCube reported the detection of $\mathrm{PeV}$ scale astrophysical neutrinos ( $v$ 's), whereas the origin has not been clearly identified yet [1]. High-energy neutrinos uniquely provide indisputable evidence for hadronic acceleration in the universe. The energy spectrum follows globally a broken $\mathrm{E}^{-\alpha}$ power law, where $\alpha=2.7 \sim 3.1$, which indicates shock acceleration. Several astronomical object classes have been proposed as potential hadronic accelerators through charged pion production in collisions with radiation fields or the ambient matter, in reactions such as: $p+\gamma \rightarrow \Delta^{+} \rightarrow \pi^{0}+p, \pi^{+}+n ; p+$ nucleus $\rightarrow \pi+X\left(\pi=\pi^{0}, \pi^{ \pm}\right)$. Subsequent decay gives the approximate neutrino flavour ratio $v_{e}: v_{\mu}: v_{\tau}=1: 2: 0$ at the sources, which is turned into the ratio of $v_{e}: v_{\mu}: v_{\tau}=1: 1: 1$ by neutrino oscillation upon arrival at Earth. The photopion $(p \gamma)$ reaction is typically the main neutrino generation process where extra galactic sources like jets and cores of active galactic nuclei (AGN) and $\gamma$-ray burst (GRB) jets have been widely studied [2, 3]. Some sources like starburst galaxies (SBGs) may emit the neutrino fluxes mainly through the hadronuclear $(p p)$ reaction [4]. In a consequence, the high pointing-back accuracy detection of $v$ 's with energies in the $\mathrm{PeV}$ range or above can lead clearly identifying the physics mechanism as well as the location of the accelerator. The GRB standard model [5], which is based on internal/external shock acceleration, has been used to describe the general features of a GRB and the observed multi-wavelength afterglow. In some of GRBs, precursor activities were observed [6]. Adding to the pointing-back of VHE $v$ 's, search for optical transients associated with very-high energy accelerators such as GRBs through the precursor and afterglow activities using the large field-of-view (FOV) of Ashra-1 can be a good probe to better understand the ambiguous acceleration mechanism.

The Earth-skimming $v_{\tau}$ technique enjoys a large target mass by detecting extensive air-showers produced by tau lepton decays in the atmosphere. The tau leptons, produced by VHE tau neutrinos that interact with the Earth matter they traverse, emerge out of a mountain or the ground facing the detector. This method has detection sensitivity in the PeV-EeV region, and can be used to search for $v_{\tau}$ 's originating from hadron acceleration in astronomical objects. Additional advantages are perfect shielding of cosmic ray secondaries, precise arrival direction determination, and negligible background from atmospheric $v$ 's [7].

\section{Ashra Experiment and Observation}

The all-sky survey high-resolution air-shower detector (Ashra) is a complex of unconventional optical collectors that image VHE air showers in a $42^{\circ}$ diameter FOV covering a large area of the night sky with a resolution of a few arcminutes [8, 9,10]. The first phase of the Ashra experiment (Ashra-1) was constructed on Mauna Loa at $3300 \mathrm{~m}$ above sea level on Hawaii Island, and includes an observatory. Ashra-1 uses electrostatic lenses 111 in addition to an optical system to generate convergent beams, enabling a very low cost and high performance image sensor, providing a high resolution over a wide FOV. The electron optics use an image pipeline to transport the image from the focal sphere of the reflective mirror optical system. After the light from the image is split, it is transported to both a trigger device and high-gain, high-resolution complementary metal-oxide semiconductor image sensor. 

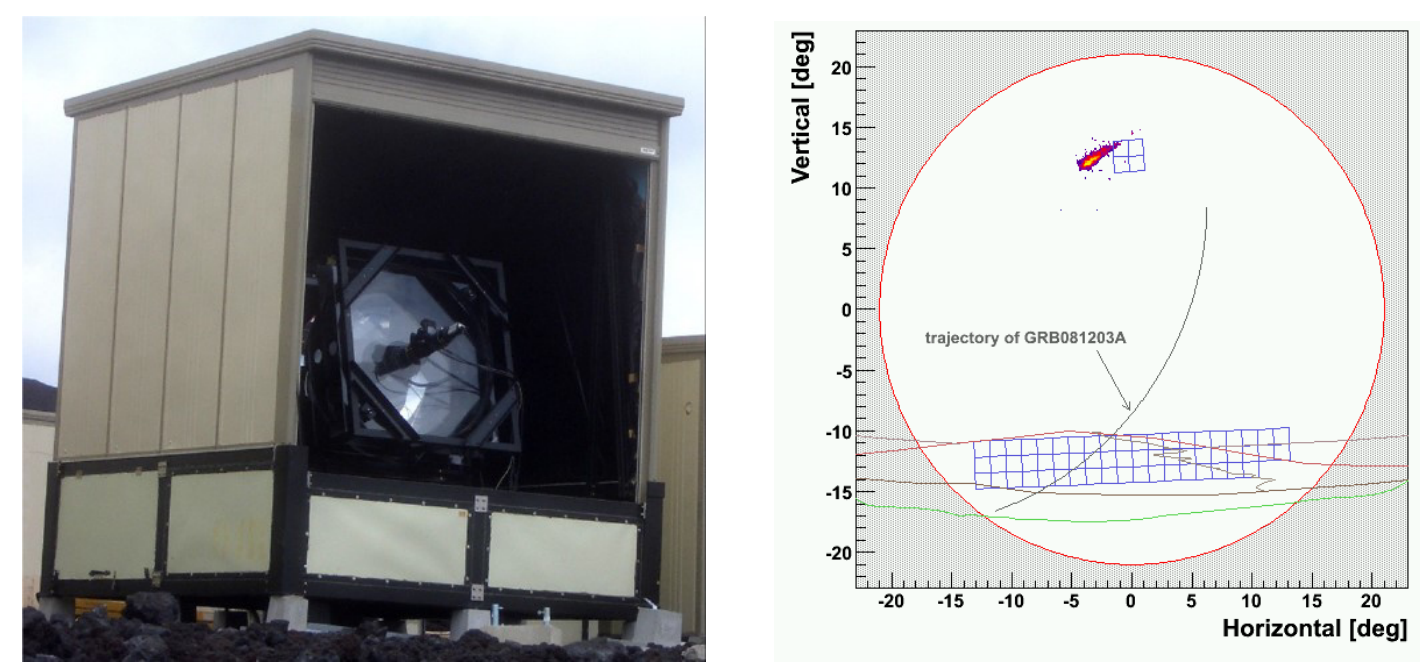

Figure 1: Left: The Ashra-1 light collector facing Mauna Kea. Right: Boundary (large red circle) between the inside (open circle) and outside (hatched area) of the FOV of the light collector and the layout of trigger pixel FOVs (blue boxes) for Cherenkov $\tau$ shower observation. Attached array of the trigger pixel FOVs (upper four blue boxes) to check the detection sensitivity with ordinary cosmic-ray air showers at a higher elevation. A simulated image of a cosmic-ray air shower readout along the trigger (points). An extended portion of the trajectory of GRB081203A counterpart (circular arc), the ridge lines of Mauna Kea (red) and Mauna Loa (green) mountains, the horizon, and Mauna Kea access road are shown.

One of the Ashra light collectors built on Mauna Loa has the geometrical advantages of not only facing Mauna Kea (Fig.11 Left), allowing it to encompass the large target mass of Mauna Kea in the observational FOV, but has also an appropriate distance of $\sim 30 \mathrm{~km}$ from Mauna Kea, yielding good observational efficiency when imaging air-shower Cherenkov lights which are directional with respect to the air-shower axis. Using the advanced features, we performed observational search for Cherenkov $\tau$ showers for $1863 \mathrm{hr}$ between January of 2012 and March of 2013. We served limited 58 channels of photomultiplier tubes (PMTs) as trigger sensors prepared for the search runs to cover the view of the surface area of Mauna Kea, maximizing the trigger efficiency for Cherenkov $\tau$ showers from Monte Carlo (MC) study, as shown in Fig. 1 Adjacent-two logic was adopted to trigger the fine imaging, by judging discriminated waveform signals from each pixel of the multi-PMT trigger sensor.

Another Ashra light collector views $42^{\circ}$ circle region, of which center points $(A l t, A z)=$ $\left(60.0^{\circ}, 0.0^{\circ}\right)$. The Ashra acquired optical images exposure for 4 seconds every 6 seconds. We have taken data over 3 observational period from 2008 to 2013, 1566(2319,1898) hours for the observation $1(2,3)$.

\section{Analysis}

To investigate the features, selection criteria, detection efficiency, and background rate for the observation of Cherenkov $\tau$ shower images, we generated $v_{\tau}$ MC events with primary energies of $1 \mathrm{PeV}$ to $100 \mathrm{EeV}$ by 0.5 decade steps, which entered into the rock of Mauna Kea uniformly and isotropically from a sufficiently large aperture. We used a geodetic database around Hawaii island 
[12], and surveyed for ourselves the position of the observatory and the terrain of the mountain and its surroundings. To study the generation and propagation of $\tau \mathrm{s}$ in the earth and in the mountain, we used PYTHIA [13] to simulate the charged current interaction of $v_{\tau} \mathrm{s}$ with nucleons and GEANT4 [14] for the energy loss due to pair production and bremsstrahlung in $\tau$ propagation. Photonuclear interaction was estimated using the differential crosssection given in [15] and [16]. TAUOLA [17] was used for $\tau$ decays and CORSIKA [18] for air showers induced by $\tau$ decays. To simulate the Ashra detector, we took into account the geometry, light collection area, mirror reflection, corrector lens transmittance, and the quantum efficiency of the photoelectric tubes, so that the fine images corresponding to the trigger judgement were simulated in an event-by-event manner. $\Delta \theta_{\tau}$, the deflection angle of $\tau$ with respect to the primary $v_{\tau}$, was estimated to be significantly less than 1 arcmin at energies above $1 \mathrm{PeV}$ due to the physical processes occurring in the rock of the earth and of the mountain [7] In addition, the reconstructed $\tau$ shower axis can point toward the $v_{\tau}$ object within an accuracy of $0.1^{\circ}$ if the resolution of the image is sufficiently high. Therefore, the Cherenkov $\tau$ shower induced by PeV-EeV $v_{\tau}$ is a fine probe into VHE hadron accelerators once an image is obtained with sufficient resolution. Such images can be obtained with the Ashra detector.

The photometric and trigger sensitivity calibration of the Ashra light collector was based on a very stable $\mathrm{YAP}\left(\mathrm{YAlO}_{3}\right.$ :Ce)-light pulser [19] which was placed at the center of the input window of the photoelectric lens image tube mounted on the focal sphere of the optical system and illuminated it. Non-uniformity in the detector gain due to the input light position was relatively corrected by mounting a spherical plate uniformly covered with luminous paint on the input window. To correct for the time variation of the photometric and trigger sensitivity because of variations in atmospheric optical thickness, which were mainly due to clouds and hazes during the observation period, we performed careful cross-calibration to compare the instrumental photoelectric response with the photometry of standard stars such as BD+75D325 of B-magnitude 9.2, for which the detected images passed through the same optical and photoelectric instruments except for the final trigger-controlled readout device. We estimated the systematic uncertainty on the basis of our understanding of the detector sensitivity to be $30 \%$ after applying a combination of the above three complementary calibration procedures.

To check the detection sensitivity and the reconstruction procedure for the Cherenkov $\tau$ shower, we detected and analyzed 824 events of ordinary cosmic-ray air-shower Cherenkov images for a total of $1884 \mathrm{hrs}$ almost simultaneously with the $v_{\tau}$ search using the same instruments used in the Ashra light collector, but triggered with the attached layout of four trigger pixels to view the sky field above Mauna Kea (Fig. 11). In the cosmic-ray observation, the trigger pixel layout is centered at zenith angle of $\sim 65^{\circ}$. Due to the directionality of the air-shower Cherenkov lights, the photometric detection of the lights $\tilde{N}_{\gamma}$ was strongly dependent upon the impact parameter $R_{P}$ in addition to the primary energy $E$. From a detailed study of MC simulated proton shower events generated with CORSIKA, we obtained a correction function to estimate the observed primary energy $\tilde{E}$ as a function of $\tilde{N}_{\gamma}$ and the long axis length $\tilde{L}$ calculated using Hillas analysis [20], where $\tilde{L}$ was used as an estimator of $R_{P}$. As a result, the total MC reconstructed energy resolution was estimated to be $62 \%$ by evaluating the rms of the $\Delta E / E=(\tilde{E}-E) / E$ distribution, for which the error was dominated by the ambiguity in $R_{P}$. The same reconstruction procedure was applied both to the observed data and the MC data. The observed and MC cosmic-ray flux spectra are shown in Fig. 2. in which the MC prediction used the typically observed cosmic-ray flux with $E>3 \mathrm{PeV}$ given by 


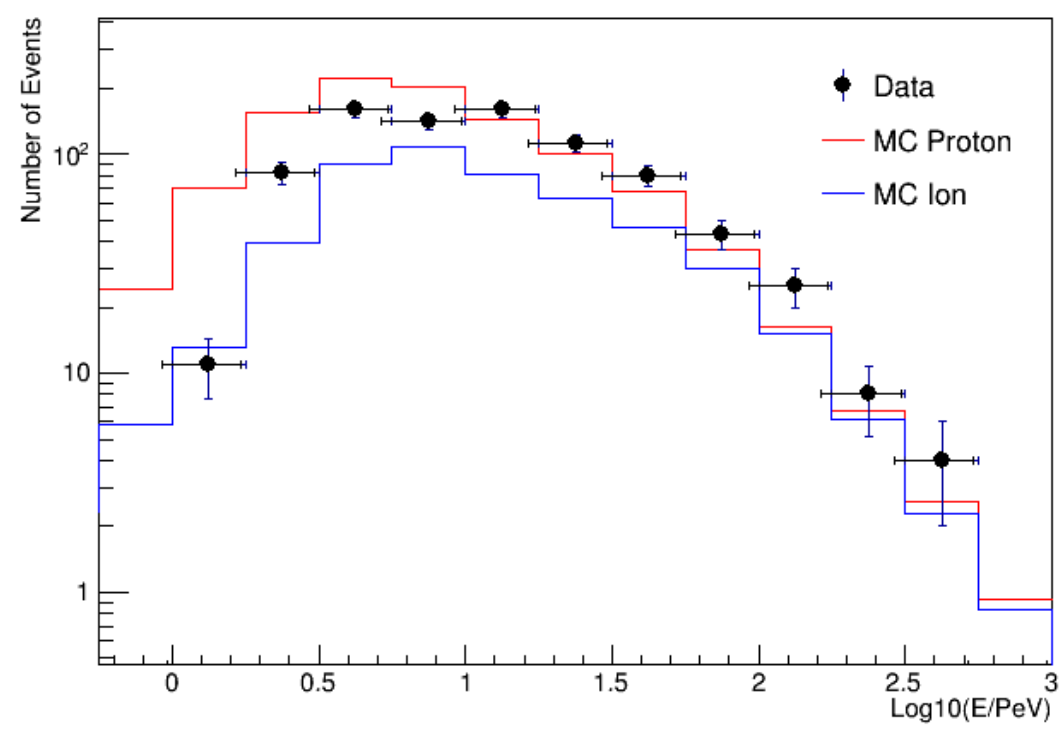

Figure 2: Observed cosmic-ray flux spectrum (filled circles) with bars indicating statistical and systematic errors and the MC predictions for proton primary (red histogram) and iron primary (blue histogram) assumptions. The width of the bands shows the evaluated systematic error of $30 \%$ of the MC prediction (see the text).

$d N / d E=(3.7 \pm 1.1) \times 10^{6} E^{-3.0}\left(\mathrm{~m}^{2} \mathrm{srs} \mathrm{GeV}\right)^{-1}$ fitting to the cosmic-ray flux observations in the knee region [21,22] with the power-law indices $-2.7(E<3 \mathrm{PeV})$ and $-3.0(E>3 \mathrm{PeV})$ assuming continuity at the knee point. We quote systematic uncertainties of $30 \%$, mainly due to cosmic-ray flux spectrum observations around the knee (20\%-28\%), and partly due to their parameterization $(\sim 14 \%)$. Since the primary cosmic-ray components are observationally undefined, we present the MC prediction of cosmic-ray flux spectra, assuming either only protons or irons as the primary cosmic rays in Fig. 2 In comparison with commisioning $v_{\tau}$ runs in the first observation period (Obs01) [23], the product of observation time and solid angle for Obs03 is larger by a factor of 2.7. According to the $\mathrm{MC}$ study, the effective detection area of $\mathrm{Obs} 03$ was 1.7 times better than that of Obs01 due to the more optimized trigger electronics. The total ratio of the observed events is expected 4.4. The observed data from $\mathrm{Obs} 03$ and the $\mathrm{MC}$ prediction agreed well on the normalization and the shape of the distribution within the expected errors. The estimation of the detection sensitivity of the Ashra light collector and the validity of the reconstruction procedure were well demonstrated.

For the $v_{\tau}$ search, we used image data acquired using the trigger for $1863 \mathrm{hrs}$ in only case of the data status defined as good out of the total observation time of $1950 \mathrm{hrs}$. To ensure the quality of the observation, we filtered the data requiring the total amount of photoelectrons $N_{p e}>250$, converted in the photoelectric lens image tube. Using a Source Extractor [24], we extracted "clusters" by requiring that of fired pixels $\left(N_{p x}\right)$ assigned to the cluster satisfied $N_{p x}>4$, where accumulated charge $\left(Q_{p x}\right)$ in the fired pixel ( $\left.p x\right)$ was required to be $Q_{p x}>6$ ADC units. The primary cluster $C$, defined as the cluster with the largest accumulated charge of fired pixels in the cluster $\left(\Sigma_{C}\right)$, was chosen as a pre-selected shower candidate for the event. To ensure that $C$ was within the effective 


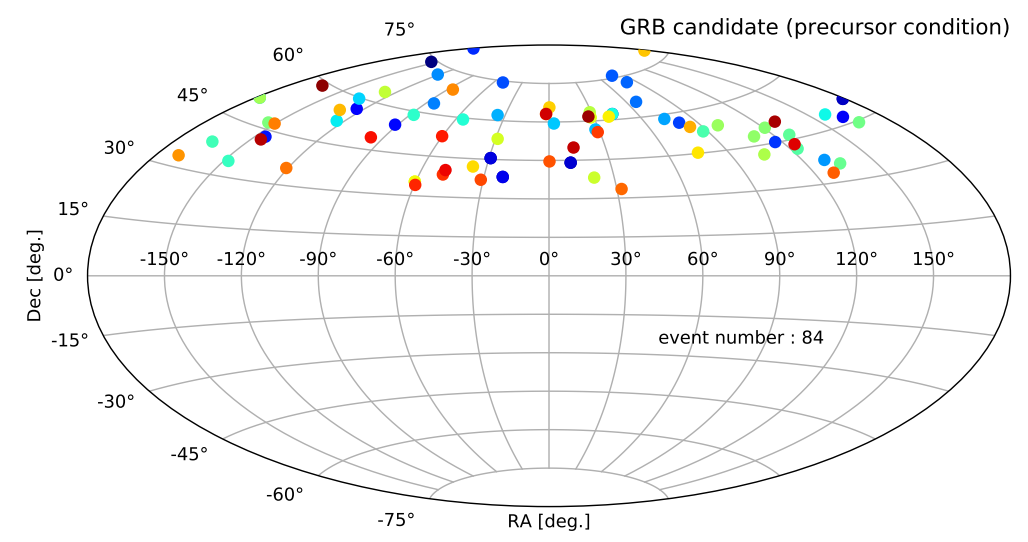

Figure 3: Precursor candidates, that is, GRB objects across the FOV prior to each trigger time, $t_{0}$, from $t_{0}-24$ to $t_{0}+1$ hours during 2008-2013 observations.

fiducial FOV, the coordinates of the center of gravity (the center of the FOV was $(0,0))\left(X_{C}, Y_{C}\right)$ of $C$ were required to satisfy $\left|X_{C}\right|<13^{\circ} .8$, and $\left(X_{C}, Y_{C}\right)$ could not overlap any obstacles such as rocks or the Mauna Loa surface within the FOV. Sometimes, "car light events" were triggered when the light beams of a car on the Mauna Kea access road entered the FOV. We determined the coordinates of the original position $\left(X_{\mathrm{car}}, Y_{\mathrm{car}}\right)$ by averaging $\left(X_{C}, Y_{C}\right)$ for events that were apparently identified as car light events in the FOV. The geometric distance $D_{\text {car }}$ in the FOV between $\left(X_{C}, Y_{C}\right)$ and $\left(X_{\text {car }}, Y_{\text {car }}\right)$ had to satisfy $D_{\text {car }}>0.2^{\circ}$. Remaining night sky backgrounds (NSBs) were rejected by requiring that $\Sigma_{C}>10 \Sigma_{C^{\prime}}$, where $C^{\prime}$ was defined as the second largest cluster. The NSB rejection was also fairly effective at removing contamination from cosmic-ray $\mu$ events, in which Cherenkov lights from cosmic-ray $\mu$ s passing through the input window glass $(8 \mathrm{~mm}$ thick) of the photoelectric lens image tube tended to separate into two or more clusters in the same image frame. In the MC study, the total selection efficiency for Cherenkov $\tau$ showers after satisfying all requirements was estimated to be $99.9 \%$ out of the filtered events with requiring $N_{p e}$ and the expected number of events of cosmic-ray $\mu$ contamination was $2.6 \times 10^{-2}$ events. We evaluated a small residual contamination of the final candidate samples from secondary particles in large angle cosmic-ray air showers of $5.7 \times 10^{-4}$ events using CORSIKA with the curved earth option. The $1863 \mathrm{hrs}$ of observation yielded a null result.

In the optical transient analysis, we search for precursors, optical emissions prior to GRB. GRB candidates are extracted from alert informations of Swift satellite and Fermi Gamma-ray Space Telescope. The time window of this search is from $t_{0}-24$ to $t_{0}+1$ hours where $t_{0}$ is GRB trigger time. We require angular distance from the center of FOV to GRB including 3 sigmas of the angle error of GRB position to be less than $21^{\circ}$ We extracted 84 (Swift:35, Fermi:49) candidates shown in Fig. 3, The precursor analysis is in progress.

\section{Results}

Fig. 4 shows the effective aperture for Cherenkov $\tau$ showers induced by $v_{\tau}$ s from sources behind the mountain and the Earth clust within the Ashra-1 light collector FOV as a function of $v_{\tau}$ energies $\left(E_{v_{\tau}}\right)$ as obtained from an MC study as changing the offline filter cuts on $N_{p e}$. On the basis 

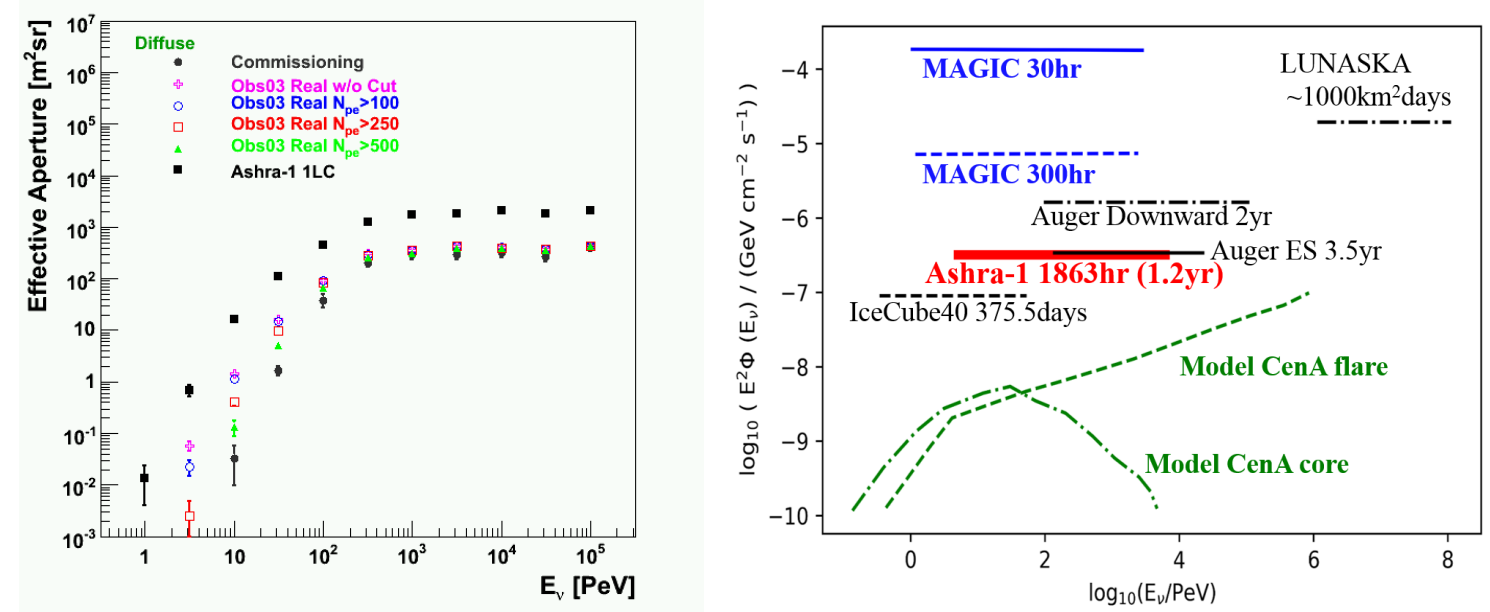

Figure 4: Left: Effective aperture for Cherenkov $\tau$ showers induced by $v_{\tau}$ s from sources behind the mountain and the Earth crust with in the Ashra-1 light collector FOV as a function of $v_{\tau}$ energy $\left(E_{v_{\tau}}\right)$, as changing the offline filtering cuts requiring the total amount of photoelectrons $N_{p e}$ in the photoelectric image tube. The improved sensitivity of the Ashra-1 light collector observation is also shown (filled squares), achieved due to halved trigger pixel size, full coverage of light collector's FOV by trigger pixels. Right: Ashra $90 \%$ C.L. limit (thick continuous line (red)) on $v_{\tau}$ flux from sources in the PeV-EeV region (see the text). For comparison, MAGIC [25], IceCube [26], Auger [27], LUNASKA [28] limits as well as model predictions 29] 30] are shown. Plots adopted from [25].

of the above null result and the estimated effective areas, we placed $90 \%$ confidence level (C.L.) upper limits: $\mathrm{E}_{v_{\tau}}^{2} \cdot \phi\left(\mathrm{E}_{v_{\tau}}\right)<3.2 \times 10^{-7} \mathrm{GeV} \mathrm{cm}^{-2} \mathrm{~s}^{-1}$ on the $v_{\tau}$ flux of sources in the PeV-EeV region, for 1863 hours observation, as shown in Fig. 4]

As sources of systematic uncertainty in the MC estimates of the effective detection areas, we considered the $v_{\tau}$ charged current interaction cross section, the energy loss by $\tau$ in the earth and the mountain, the geological model around the observation site, and the gain calibration of the light collector, which were evaluated to be $50 \%, 50 \%, 10 \%$, and $30 \%$, respectively [7]. The conservatively combined systematic sensitivity error was obtained from the square sum of the above uncertainties as $77 \%$, which affects the result of the $90 \%$ C.L. upper limit shown in Fig. [4 where we assumed a typical $E_{v}^{-2}$ flux to ensure unbiased constraints on observationally undefined physical mechanisms of GRBs and AGN flares. There are several models which predict VHE $v$ flux during our observation periods. Detailed discussions on physics interpretation from the comparison of our results with theoretical models will be made in a paper that is to be published soon. For comparison, Fig. 4 shows other observational limits on the $v$ fluxes from point sources with MAGIC [25], IceCube [26], Auger [27], LUNASKA [28] as well as theoretical model predictions [29, 30].

Our results are the most stringent in the $\mathrm{PeV}-\mathrm{EeV}$ region and complementary to other observational results for the sub-PeV and over-EeV energy regions, and indicate the advanced sensitivity of the system. Our search for PeV-EeV $v_{\tau}$ reported in this Letter complements other experiments in energy range and methodology. 


\section{Acknowledgement}

The Ashra Experiment was supported by the Coordination Fund for Promoting Science and Technology and by a Grant-in-Aid for Scientific Research and Grant-in-Aid for Scientific Research from the Ministry of Education, Culture, Sports, Science and Technology in Japan.

\section{References}

[1] M. Aartsen et al., PRL 113, 101101 (2014).

[2] for example: K. Murase it et al. ApJ Lett., 651:L5, 2006.

[3] for example: A. Neronov, and M. Ribordy, Phys. Rev. D, 80 (2009) 083008.

[4] A. Loeb and E. Waxman. Journal of Cosmology and Astroparticle Physics, 2006(05):003, 2006.

[5] Mésźaros, P. 2006, Rep. Prog. Phys., 69, 2259

[6] Burlon, D., et al. 2008, ApJ Lett., 685, L19

[7] Y. Asaoka and M. Sasaki. Astroparticle Physics, 41:7-16, 2013.

[8] Sasaki, M. 2008, J. Phys. Soc. Japan, 77SB, 83

[9] Aita, Y., et al. 2008a, Proc. 30th ICRC, 3, 1405

[10] Sasaki, M., et al. 2008, Proc. 30th ICRC, 3, 1559

[11] Asaoka, Y., \& Sasaki, M. 2011, Nucl. Instrum. Methods Phys. Res. A, 647, 34

[12] Mooney, W. D., Laske, G., \& Masters, T. G. 1998, J. Geophys. Res. , 103, 727

[13] Sjöstrand, T., Edén, P., Friberg, C., Lönnblad, L., Miu, G., Mrenna, S., \& Norrbin, E. 2001, Comput. Phys. Commun., 135, 238

[14] Agostinelli, S., et al. 2003, Nucl. Instrum. Methods Phys. Res. A, 506, 250

[15] Iyer Dutta, S., Reno, M. H., Sarcevic, I., \& Seckel, 2001, Phys. Rev. D, 63, 094020

[16] Abramowicz, H., \& Levy, A. 1997, arXiv:hep-ph/9712415v2

[17] Jadach, S., Wass, Z., Decker, R., \& Kühn, J. H. 1993, Comput. Phys. Commun., 76, 361

[18] Heck, D., Schatz, G., Thouw, T., Knapp, J., \& Capdevielle, J. N. 1998, Report FZKA 6019

[19] Kachanov, V. A., et al. 1992, Nucl. Instrum. Methods Phys. Res. A, 314, 215

[20] Hillas, A. M. 1985, Proc. 19th ICRC, 3, 445

[21] Antoni, T., et al. 2005, Astropart. Phys., 24, 1

[22] Amenomori, M., et al. 2008, ApJ, 678, 1165

[23] Y. Aita, et al., ApJL 736, L12 (2011).

[24] Bertin, E., \& Arnouts, S. 1996, A\&AS, 117, 393

[25] M. L. Ahnen, et al., Astroparticle Physics 102, 77 (2018).

[26] R. Abbasi, Y. Abdou, T. Abu-Zayyad, et al., ApJ, 732 (2011) 18.

[27] P. Abreu, et al. 2012, ApJ Lett., 755 (2012) L4

[28] C.W. James, R.J. Protheroe, R.D. Ekers, et al., MNRAS, 410 (2011) 885.

[29] A. Cuoco, S. Hannestad, Phys. Rev. D, 78 (2008) 023007.

[30] M. Kachelriess, S. Ostapchenko., and R. Tomas, New J. Phys., 11, (2009) 065017. 